\title{
MAGNETIC PROPERTIES OF GLASSES WITH HIGH IRON OXIDE CONTENT
}

\author{
Maximina Romero, Jesús Ma. Rincón \\ Laboratorio de Materiales Vitrocerámicos. Instituto E. Torroja de Ciencias de la \\ Construcción (CSIC), Madrid, Spain \\ Carlos J.R. González Oliver \\ CONICET - Instituto Balseiro. San Carlos de Bariloche, Argentina \\ Carlos D’Ovidio, Daniel Esparza \\ Centro Atómico Bariloche and Instituto Balseiro (CNEA). San Carlos de Bariloche, \\ Argentina
}

\begin{abstract}
$\underline{\text { Abstract }}$
A study of the magnetic properties of glasses with high iron oxide content has been carried out. This glass series was obtained by recycling goethite $(\mathrm{FeOOH})$ industrial waste, with dolomite and glass cullet as complementary raw materials. The magnetisation as a function of applied magnetic field for these glasses was measured. According to the magnetic behaviour, which closely correlates with the iron content, these glasses can be classified as ferrimagnetic, superparamagnetic and paramagnetic. The magnetisation curves were analysed in terms of current models for these types of magnetic behaviour.
\end{abstract}

Keywords: A. glasses, A. magnetic materials, D. magnetic properties

\section{Introduction}

The possibility of vitrifying industrial wastes has been recently investigated not only for immobilising toxic components, but also for recycling to produce new materials. With this purpose, research focused on the production of new glasses and glass-ceramics from goethite $(\mathrm{FeOOH})$ industrial wastes has been carried out [1]. The waste, which is in the form of a red mud that comes from the zinc process, contains besides goethite, large quantities of lead and zinc oxides [2]. This paper presents a study of the magnetic properties of glasses made from goethite wastes combined with glass forming materials, which are also wastes from other industries. The results show a series of glasses with different iron oxide content and interesting magnetic properties. Due to the insulating character of these materials and their magnetic properties they could be candidates for very high frequency applications.

\section{Experimental}

Goethite industrial wastes were combined with dolomite industrial wastes and soda-lime glass cullet in different proportions to obtain glasses with increasing iron oxide concentration. Prior to melting, the powdered raw materials were mixed in a ball mill for 10 minutes. The precursors were melted in aluminous fireclay crucibles in a Superkanthal electric furnace (SWEDISH AB pob 505 model), at $1450^{\circ} \mathrm{C}$ for 30 minutes. The melts were poured into brass 
moulds and annealed at $500^{\circ} \mathrm{C}$ for 2 hours. Disks of $4 \mathrm{~mm}$ diameter and $0.5 \mathrm{~mm}$ thickness were cut from the bulk pieces for magnetic measurements.

The chemical composition of the glasses is shown in Table 1 . The analyses were done by ICP (Inductively Coupled Plasma) and converted to oxide weight percent, with an error of about $0.1 \%$. Iron oxide is expressed as $\mathrm{Fe}_{2} \mathrm{O}_{3}$, but it can also be present as $\mathrm{FeO}$, as well as $\mathrm{Fe}_{3} \mathrm{O}_{4}$. The glasses were labelled according to the iron content in decreasing order. The other oxide concentrations are somewhat random due to the different proportions employed for the raw materials, as this was primarily intended for glass forming studies.

Table 1. Chemical composition of the glasses

\begin{tabular}{lccccccccc}
\cline { 2 - 7 } & $\mathrm{Fe}_{2} \mathrm{O}_{3}$ & $\mathrm{SiO}_{2}$ & $\mathrm{Al}_{2} \mathbf{O}_{3}$ & $\mathrm{CaO}$ & $\mathrm{MgO}$ & $\mathrm{ZnO}$ & $\mathrm{PbO}$ & $\mathrm{Na}_{2} \mathbf{O}$ & $\mathrm{K}_{2} \mathbf{O}$ \\
\hline G1 & 29.2 & 38.4 & 6.4 & 6.6 & 2.9 & 9.5 & 2.2 & 3.9 & 0.8 \\
G2 & 25.8 & 43.5 & 4.7 & 7.0 & 3.3 & 8.3 & 1.8 & 4.8 & 0.7 \\
G3 & 25.0 & 40.8 & 8.1 & 8.7 & 4.0 & 7.3 & 1.6 & 3.6 & 0.9 \\
G4 & 23.4 & 39.5 & 10.8 & 9.5 & 4.6 & 7.0 & 1.7 & 2.5 & 1.0 \\
G5 & 18.6 & 37.5 & 7.6 & 17.5 & 8.6 & 5.3 & 1.1 & 2.9 & 0.9 \\
G6 & 18.4 & 41.3 & 12.4 & 11.5 & 5.7 & 5.6 & 1.2 & 2.6 & 1.2 \\
G7 & 18.0 & 44.9 & 3.6 & 14.0 & 6.6 & 5.3 & 1.3 & 5.7 & 0.6 \\
G8 & 16.4 & 50.1 & 3.7 & 10.8 & 5.4 & 5.0 & 1.4 & 6.7 & 0.6 \\
G9 & 15.6 & 53.9 & 2.1 & 8.8 & 4.1 & 5.1 & 1.5 & 8.4 & 0.4 \\
\hline
\end{tabular}

Magnetisation curves were measured in a vibrating sample magnetometer (VSM) [3], at room temperature and in fields up to $10 \mathrm{KOe}$. The applied magnetic field, along a sample diameter, was parallel to the vibrating direction. No correction for the applied magnetic field was necessary because the demagnetising factor by magnetisation product was low, even at the highest magnetisation. The magnetisation values were normalised by the sample weight, using the magnetic moment per gram or emu/g as magnetisation unit.

\section{$\underline{\text { Results and Discussion }}$}

In Figure 1 representative XRD patterns of the glasses are shown. Glass G1 presents diffraction peaks that correspond to magnetite, indicating crystallisation of some of the iron oxide. Glasses G2, G3 and G4 showed incipient peaks at d = 2.52 A, that can be attributed to magnetite. Glasses G5 to G9 had amorphous character. 


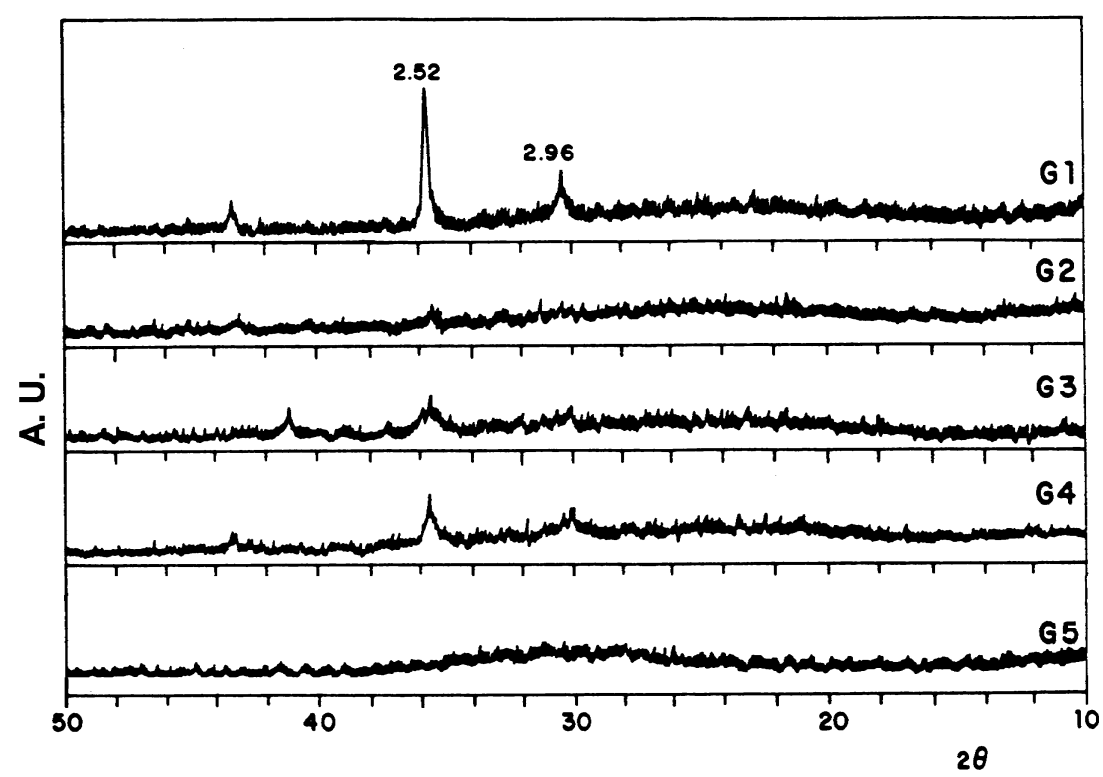

Figure 1. Representative XRD patterns of the glasses studied

Figures 2, 3 and 4 show the magnetisation vs. applied field curves. The complete hysteresis loop was reduced to the first quadrant for clarity and to restrict the numerical analysis to positive values.

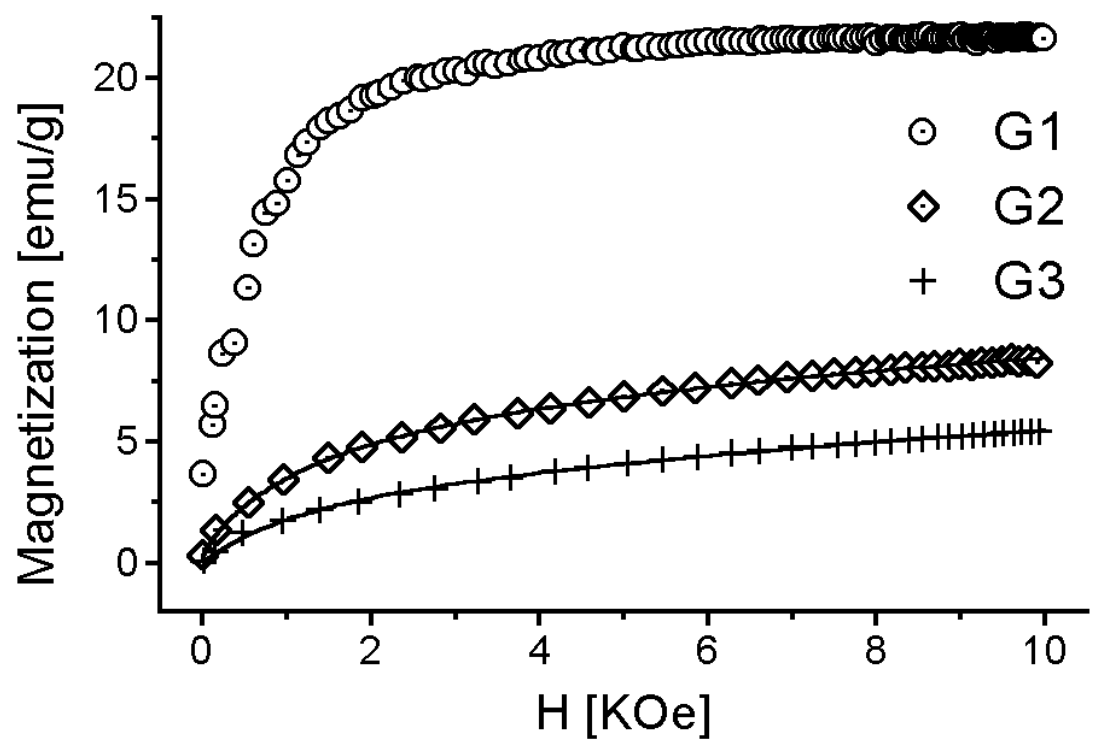

Figure 2: Magnetisation curves for glasses G1, G2 and G3. The continuous lines represent the fitting using equation (1). For clarity only one tenth of the experimental points are shown. 


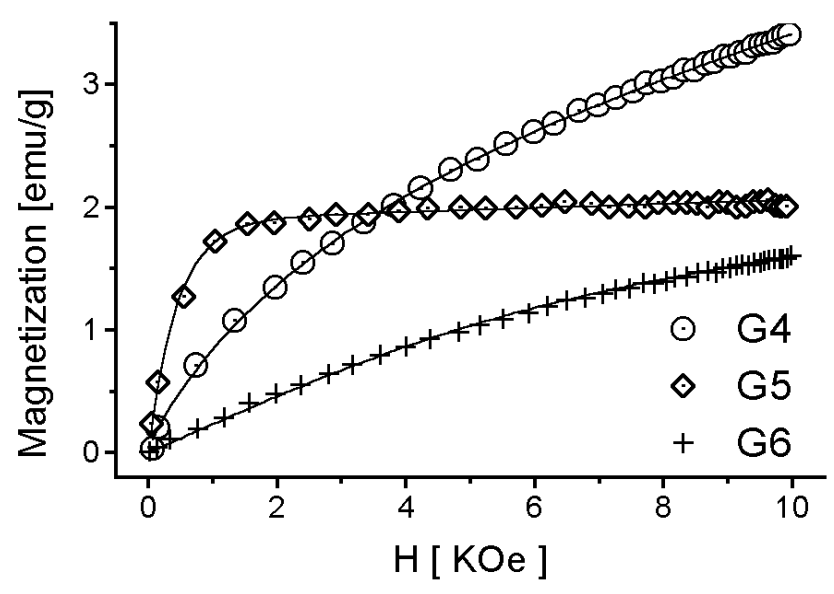

Figure 3: Magnetisation curves for glasses G4, G5 and G6. The continuous lines represent the fitting using equation (1). For clarity only one tenth of the experimental points are shown

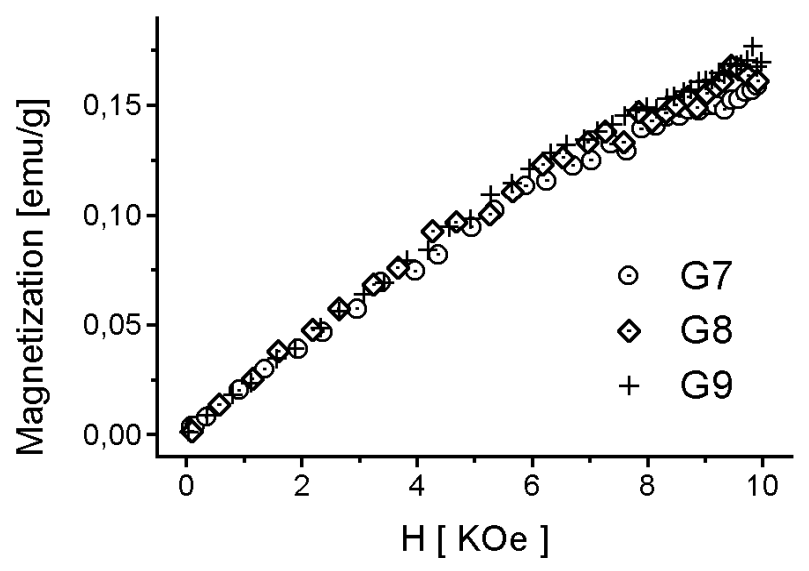

Figure 4: Magnetisation curves for glasses G7, G8 and G9. For clarity only one tenth of the experimental points are shown.

In a first qualitative analysis, it can be observed that glasses G1 and G5 have magnetisation curves that saturate at low fields, characteristic of ferromagnetic or ferrimagnetic materials. For glasses G2, G3, G4 and G6, the magnetisation increases slowly with the applied field and tends to saturate at moderate fields. This behaviour is typical of materials known as superparamagnetics. Finally, for G7, G8 and G9 glasses, the magnetisation increases almost linearly with applied field up to moderate fields, characteristic of paramagnetic materials.

Table 2 collects some experimental magnetic parameters and some calculated parameters that arise from standard theoretical models of magnetism. $\chi_{0}$ is the experimental magnetic susceptibility for $\mathrm{H}=0$, equal to the linear term coefficient of a polynomial fit of the complete magnetisation curve between $\mathrm{H}=-1000$ Oe and $\mathrm{H}=+1000$ Oe. $\mathrm{M}_{10}$ is the magnetisation value at $10 \mathrm{~K}$ Oe that, although it is not generally the saturation value, serves as a reference. 
The $\mathrm{M}_{10}$ and $\chi_{0}$ values decrease with iron oxide content. Table 2 also includes the iron oxide content for comparison.

Table 2. Experimental and calculated magnetic parameters of the glasses

\begin{tabular}{ccccccc}
\hline $\begin{array}{c}\mathbf{F e}_{2} \mathbf{O}_{3} \\
{[\%]}\end{array}$ & $\begin{array}{c}\chi_{\mathbf{0}} \\
(\mathrm{e} u . O e / g)\end{array}$ & $\begin{array}{c}\mathbf{M}_{\mathbf{1 0}} \\
(\mathrm{emu} / \mathrm{g})\end{array}$ & $\begin{array}{c}\chi \\
(\mathrm{emu} . \mathrm{X} / \mathrm{g})\end{array}$ & $\begin{array}{c}\mathbf{X} \\
----\end{array}$ & $\begin{array}{c}\mu \\
----\end{array}$ & $\begin{array}{c}\lambda \\
(\text { Oe.g/emu })\end{array}$ \\
\hline 29.2 & $3.110^{-2}$ & 21.5 & ---- & ---- & ---- & ---- \\
25.8 & $6.310^{-3}$ & 8.49 & $7.410^{-5}$ & $1.710^{18}$ & 680 & 1600 \\
25.0 & $3.210^{-3}$ & 5.44 & $1.210^{-4}$ & $1.110^{18}$ & 580 & 3200 \\
23.4 & $1.210^{-3}$ & 3.11 & $1.110^{-4}$ & $6.910^{17}$ & 540 & 5700 \\
18.6 & $3.310^{-3}$ & 2.05 & ---- & ---- & ---- & ---- \\
18.4 & $2.610^{-4}$ & 1.61 & $2.910^{-5}$ & $1.410^{18}$ & 220 & 16500 \\
18.0 & $2.110-5$ & 0.15 & ---- & ---- & 4.6 & ---- \\
16.4 & $3.210^{-5}$ & 0.17 & ---- & ---- & 6.1 & ---- \\
15.6 & $2.710^{-5}$ & 0.17 & ---- & ---- & 5.7 & ---- \\
\hline
\end{tabular}

Glass G1 has the highest iron oxide concentration and reaches the highest value of magnetisation of all the samples, following a curve that saturates at low field. This can be explained assuming that iron oxide precipitates as magnetite $\left(\mathrm{Fe}_{3} \mathrm{O}_{4}\right)$, which is ferrimagnetic. In general, the iron atoms of these glasses can be present in two different states: as a solid solution or as a precipitated second phase plus a solid solution. In the first case, there is homogeneity at atomic level and in dilute systems a large separation between iron atoms is expected, with little or no interaction between them. In the other case, the presence of precipitates indicates that a solubility limit has been reached. Part of the iron oxide can be found as particles of different size and distribution, the rest of the oxide remaining dissolved in the glass matrix. When the precipitates are big enough to exhibit full magnetic order, the material behaves proportionally as bulk ferrimagnetic magnetite, as found for glass G1.

A simple calculation indicates that the observed saturation magnetisation of sample G1 is equivalent to $83 \%$ of the iron oxide being as magnetite. The remnant $17 \%$ would be as a solid solution and would contribute to magnetisation only at extremely high field. Previously [4] it was shown, by X-ray diffraction and Mössbauer spectroscopy that glass G1 had crystallised extensively during cooling having approximately $73 \%$ of the iron oxide as magnetite, in agreement with this calculation.

Glasses G2, G3, G4 and G6 behave as superparamagnets. This behaviour has been observed in partially reacted ferrites, in $\mathrm{Mn} \mathrm{Cu} \mathrm{Al}$ alloys and in magnetite dispersions in a silicate matrix [5]. It comes from the interaction of giant magnetic moment clusters weakly coupled by a Weiss mean field. Beck [6] describes the superparamagnetic behaviour as the sum of a susceptibility term and a Brillouin function term with mean field as follows: 


$$
M=\chi \cdot H+\mu_{B} \cdot X \cdot\left[(\mu+1) \cdot \operatorname{coth}\left[\mu_{B} \cdot(\mu+1) \cdot A\right]-\operatorname{coth}\left[\mu_{B} \cdot A\right]\right]
$$

with:

$$
A=\frac{H+\lambda \cdot(M-\chi \cdot H)}{K \cdot T}
$$

where $\mathrm{M}$ is the magnetisation, $\mathrm{H}$ is the applied field, $\chi$ is the residual susceptibility of the matrix at high fields, $\mathrm{X}$ is the number of clusters, $\mu$ is the total number of Bohr magnetons for each cluster, $\lambda$ is the Weiss molecular field constant, $K$ is the Boltzmann constant, $\mu_{B}$ is the Bohr magneton and $\mathrm{T}$ is the absolute temperature.

The magnetisation curves of glasses G2, G3, G4 and G6 were fitted with equation (1) and the parameters were found by least square fitting. The best-fit curves are included in Figures 2, 3, and the fitting parameters in Table II. From the numerical analysis, equation (1) is overparametrized; consequently, there is a strong dependence between parameters and the fitting errors are large, in the order of $10 \%$. It is not possible to reduce the number of parameters because they all have independent physical meaning. The values of $\chi, X, \mu$ and $\lambda$ can be interpreted as follows. The $\mu$ values decrease monotonically with iron content. The residual susceptibility $\chi$ has values around $10^{-4}$ for G2, G3 and G4 while for G6 it approaches the $\chi_{0}$ values found for G7, G8 and G9. It indicates that the superparamagnetic clusters are immersed in a matrix having in turn paramagnetic atoms for G7, G8 and G9 glasses. The number of clusters X, decreases from G2 to G4 and increases again for G6, suggesting that there are more clusters with less magnetic moment. Surprisingly $\lambda$ increases as the iron oxide content of the glasses decreases, which could be related to a larger quantity of smaller clusters (as in G6) that became closer, thus increasing the magnetic interaction.

The Brillouin function in equation (1) tends to a magnetisation value equal to X. $\mu_{\mathrm{B}} \mu$ as $\mathrm{H}$ tends to infinity. These values are 10.8, 6.18, 3.47 and 2.86 emu/g for G2, G3, G4 and G6 glasses respectively, using the values of Table II. These values are much lesser than those calculated assuming that all the iron atoms have a magnetic moment of $5 \mu_{\mathrm{B}}\left(\mathrm{Fe}^{3+}\right)$ and are aligned in an infinite magnetic field: 90.1, 87.6, 81.8 and $64.4 \mathrm{emu} / \mathrm{g}$ respectively. This indicates that the magnetisation due to clusters involves only a small portion of the iron atoms; the rest of the iron contributes from the matrix in the paramagnetic state only at extremely high magnetic fields. A similar analysis could be done assuming that all the iron atoms are combined as magnetite yielding values of: 22.8, 22.1, 20.7 and $16.3 \mathrm{emu} / \mathrm{g}$ respectively. Again, cluster magnetisation cannot account for the total iron of the samples. Equation (1) can be reduced for $\mathrm{H} \rightarrow 0$ to:

$$
M=\frac{N \mu^{2} \mu_{B}{ }^{2}}{3 K_{B} T} H=\chi_{0} H
$$

where $\mathrm{N}$ is the number of magnetic atoms with moment $\mu . \mu_{\mathrm{B}}$ and $\chi_{0}$ is the experimental initial susceptibility. This linear dependence of $\mathrm{M}$ on $\mathrm{H}$ can be observed for G7, G8 and G9 in Figure 3. Using equation (2), we have calculated the magnetic moment $\mu_{\text {. }} \mu_{\mathrm{B}}$ of each iron atom, whose number $\mathrm{N}$ has been deduced from the composition. The values of $\mu$ corresponding to these glasses, Table II, are close to 5, which is the value for trivalent iron. 
These results are consistent with those obtained previously [4] by Mössbauer spectroscopy: about $80 \%$ of the total iron is as $\mathrm{Fe}^{3+}$. Therefore, these glasses behave as paramagnets with isolated iron atoms as the source of magnetic moment. This means that the iron compounds, that carry the magnetic iron ions, are in solid solution in the glass matrix and that their concentration is not enough for the appearance of precipitates. Consequently, we can estimate a solubility limit as approximately $18 \% \mathrm{Fe}_{2} \mathrm{O}_{3}$ concentration. This result is consistent with the previously calculated non-crystallised iron oxide remnant of G1 glass.

G5 glass is anomalous as it presents ferrimagnetic type behaviour in spite of its iron concentration that is at the limit between superparamagnetic and paramagnetic response. However, the magnetisation value at $10 \mathrm{~K}$ Oe $\left(\mathrm{M}_{10}\right)$ is in the appropriate range (see Figure 3). This behaviour can be due to a reduction of the iron oxide solubility provoking an anticipated precipitation of big clusters with ferrimagnetic order. Coincidentally G5 glass has the highest calcium oxide and magnesium oxide concentration and the lowest silica concentration.

The composition of the glassy matrix is not the same for the different samples investigated here and consequently this could influence the solubility limit of some species and the size and distribution of any precipitates. However, from this analysis, the magnetic properties correlate fairly well with the iron oxide content, except for G5 glass. We will continue this work with synthetic glasses having a simpler and constant glass matrix composition and different amounts of added iron oxide. We will also extent the measurement range to lower temperatures and higher magnetic fields.

\section{Conclusions}

The magnetic properties of several glass samples with different iron oxide content have been studied. At low iron oxide contents, we observed paramagnetic behaviour with values of atomic moment close to $5 \mu_{\mathrm{B}}$ corresponding to trivalent iron $\left(\mathrm{Fe}^{3+}\right)$. At the highest iron oxide concentration, we found ferrimagnetic behaviour, where about $83 \%$ of the iron oxide is precipitated as magnetite while the rest of the oxide remains in solid solution. Above $18 \%$ $\mathrm{Fe}_{2} \mathrm{O}_{3}$, we observed a mixed behaviour: precipitated magnetic clusters contribute to a superparamagnetic response while dissolved iron atoms behave paramagnetically and contribute to the magnetisation at very high magnetic fields.

The magnetic properties found correlate fairly well with the iron oxide content of this glass series.

\section{Acknowledgements}

The authors thank the European Union (European Project RAW MATERIALS DG-XII (MA 2R CT90-0007)) for partial financial support of this work and Dr. Maria Teresa Malachevsky for the critical review of the manuscript and useful discussions. 


\section{References}

1. M. Romero, Procesado y caracterización de nuevos vidrios y materiales vitrocerámicos obtenidos por reciclado de residuos industriales de goethita. Ph.D. Thesis. Universidad de Alcalá de Henares, Facultad de Ciencias Químicas, Madrid (1995).

2. M. Romero and J.Ma. Rincón, Materials Letters, 31 (1997) 67-73.

3. J.L. Giordano and D. Esparza, Meas.Sci.Technol., 5 (1994) 509-513.

4. M. Romero and J.Ma. Rincón, J.Eur.Ceram.Soc., 18 (1997) 153-160.

5. P. Auric, N. Dang, A.K. Bandyopadhyay and J. Zarzycki, J.Non-Cryst.Solids., 50 (1982) 97-106.

6. P.E. Beck, Progress in Material Science, 23 (1978) 1-49. 